\title{
Die Bildung des Spaltöffnungsmusters in der Blattepidermis
}

\author{
(Mit Anmerkungen über weitere Musterbildungen) \\ Von Erwin Bünning und Herta Sagromsky \\ Aus dem Botanischen Institut der Universität Tübingen \\ (Z. Naturforschg. 3 b, 203-216 [1948]; eingegangen am 27. März 1948)
}

\begin{abstract}
Die Verteilung der Spaltöffnungen in der Epidermis ist-auch dann keine Zufallsverteilung, wenn diese Spaltöffnungen nicht im Zuge einer bestimmten Teilungsfolge an so prädestinierten Stellen entstehen. Vielmehr bildet jede Spaltöffnung um sich eine Hemmzone, in der keine weiteren Spaltöffnungen entstehen können. Die Intensität der Hemmung nimmt mit zunehmender Entfernung von einer Spaltöffnung ab.

Die Untersuchungen begründen folgende Vorstellung: Die differentielle Teilung, die zur Bildung der Spaltöffnungsinitiale führt, kann in der Epidermis erst eintreten, wenn die Teilungsfähigkeit fast erloschen ist, wenn also offenbar ein Teilungshormon annähernd verbraucht ist. Die so entstandene Initiale, aus der entweder eine Spaltöffnung oder auch ein Hàar entstehen kann, erwirbt mit der differentiellen Teilung mehrere neue Fähigkeiten; eine dieser Fähigkeiten führt auch zur Beeinflussung der Umgebung, es wird nämlich ein Teilungsstoff an die umgebenden Epidermiszellen abgegeben. Der Stoff kann in diesen umgebenden Zellen zu neuen Zellteilungen führen (Nebenzellbildung); auch in größerer Entfernung, wo die Konzentration nicht mehr zur Auslösung neuer Teilungen genügt, ist die Anwesenheit dieser Stoffe noch an ihrer positiv chemotaktischen Wirkung auf die Zellkerne feststellbar. Der von den Initialen abgegebene Teilungsstoff stellt in dieser Umgebung wieder den gleichen Zustand her, wie er in einer jüngeren Epidermiszelle herrschte, daher können in diesem Bereich ebenso wie in der jungen Epidermis nicht die differentiellen Teilungen stattfinden, die zur Bildung der Spaltöfnungsinitialen notwendig sind.

Die differentielle Teilung selber vollzieht sich so, daß sich das Plasma in der Zelle lokal anhäuft und die neue Wand, deren Entstehung von der Oberfläche der Plasmaanhäufung ausgeht, diese Anhäufung dann von der übrigen, größeren, aber viel plasmaärmeren Zelle abgrenzt.

Wenn sich die Epidermis vom Mesophyll lokal ablöst, kann eine Initiale ihre Hemmwirkung in diesem Gebiet nicht ausüben, so daßs dann (wie etwa bei mehreren BegoniaArten) Gruppen von Spaltöffnungen entstehen.

Den bei der Bildung der Spaltöffnungsinitialen und des Spaltöffnungsmusters geltenden Gesetzen kommt offenbar eine große Bedeutung bei den verschiedensten Teilvorgängen der pflanzlichen Gewebedifferenzierung zu.
\end{abstract}

$\mathrm{E}^{\mathrm{i}}$ in Grundproblem der pflanzlichen Differenzierung entspringt der Tatsache, daß aus genotypisch gleichen Zellen eines ursprünglich homogenen Gewebes verschiedenartige Zellen entstehen. Diese Verschiedenartigkeit der Entwicklung ist nur unter der Annahme zu verstehen, daß die Zellen wenigstens in einem bestimmten Zeitpunkt ihrer Entwicklung eine hohe Labilität besitzen, die sie befähigt, schon auf geringe Schwankungen der auf sie einwirkenden Faktoren stark zu reagieren. Solche Schwankungen modifizierender Außenfaktoren oder innerhalb der Zelle selber liegender Bedingungen sind uns als wichtige Ursache der biologischen Variabilität bekannt. Das Besondere bei der qualitativen Differenzierung besteht nur darin, daß die geringen Schwankungen der Bedingungen nicht ein fluktuierendes, sondern ein alternatives Reagieren zur Folge haben. Wir müssen also in den embryonalen Zellen einen „Kippvorgang“ annehmen, der es ermöglicht, daß diese Zellen von mehreren möglichen, qualitativ verschiedenen Entwicklungsrichtungen die eine oder die andere einschlagen. Beispielsweise kann so aus einer jungen Epidermiszelle des Blattes nach dem Abschluß der Zellteilungen entweder eine gewöhnliche Epidermiszelle entstehen, oder die Zelle zeigt nochmals eine Teilung besonderer Art, nämlich eine Teilung, die zur Abschnürung einer Initiale für eine Spaltöffnung führt. Darüber, ob dieser oder jener Weg eingeschlagen wird, kann also (wenn auch durchaus nicht in allen Fällen) der Zufall (in dem Sinne wie bei 
der Modifikabilität) entscheidend sein, und so können im Gewebe qualitativ verschiedene Elemente entstehen.

Die Bildung von Mustern im Gewebe hat aber noch eine besondere Seite. Jene Differenzierung, die Entscheidung über die Entwicklungsrichtung, also die Entscheidung darüber, ob bzw. in welcher Richtung der „Kippvorgang“ eintritt, erfolgt nicht, wie man allein auf Grund des oben skizzierten Mechanismus erwarten sollte, rein nach $\mathrm{Zu}$ fallsgesetzen. Das fertige Muster hat zum mindesten sehr häufig nicht den Charakter der Zufälligkeit, sondern den einer mehr oder weniger großen Regelmäßigkeit, d.h. die Abstände zwischen Zellen bzw. Zellgruppen, die eine gleichartige Entwicklungsrichtung eingeschlagen haben, die also den „Kippvorgang“ im gleichartigen Sinne durchgeführt haben, sind nicht beliebig, tragen nicht einen Zufallscharakter, sondern sind mehr oder weniger gleichmäßig.

Beispiele für Muster, die in diesem Sinne regelmäßig sind, lassen sich leicht anführen. Erwähnt sei etwa die regelmäßige Verteilung von Gefäßbündeln im Sproß, das regelmäßige Muster der Markstrahlinitialen im Kambium oder die regelmäßige Verteilung von Spaltöffnungen in der Epidermis (vgl. auch Hinweise bei $\mathrm{Jost}^{1}$ ).

\section{Die Regelmäßigkeit des Spalt- öf fingsmusters}

Das letztgenannte Beispiel, die Bildung des Spaltöffnungsmusters, war Gegenstand unserer Untersuchung. Wir sehen dabei natürlich von solchen Fällen ab, in denen sich die Regelmäßigkeit dieses Musters, wie namentlich bei Monokotylen, einfach daraus erklärt, daß die Spaltöffnungsinitiale jedesmal im Zuge einer ganz bestimmten Teilungsfolge entsteht. Wir beschränken uns vielmehr auf die Fälle, in denen die Spaltöffnungsinitialen durch Einschiebung einer Wand in den Ecken jeder beliebigen Epidermiszelle entstehen können.

Wenn wir hier den erstgenannten Weg der Bildung des Spaltöffnungsmusters, also die Bildung des absolut regelmäßigen Musters vieler Monokotylen zufolge eines festen Teilungsschemas, auch nicht berücksichtigen wollen, so soll doch noch das Gemeinsame beider Wege herausgestellt werden. Es besteht darin, daß die Tei-

${ }^{1}$ L. Jost, Z. Bot. 25, 481 [1932]. lung, die die Initiale entstehen läßt, erst eintritt, nachdem die übrige Teilungstätigkeit ganz oder vorübergehend (nämlich bis zur evtl. Nebenzell-

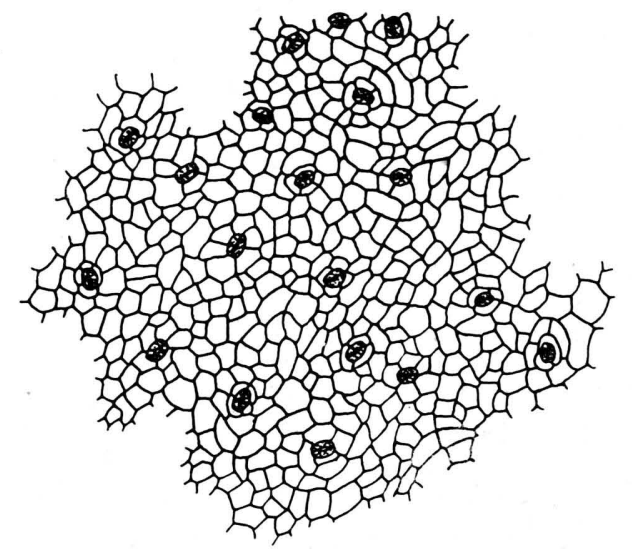

Abb. 1. Spaltöffnungsverteilung bei Bougainvillea spectabilis.

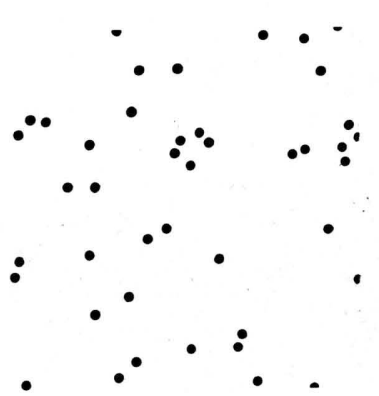

Abb. 2. Darstellung einer reinen Zufallsverteilung.
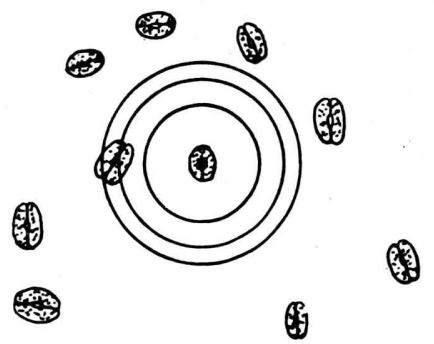

(3)

Abb. 3. Spaltöffnungsverteilung in einem Epidermisausschnitt von Sambucus nigra. Nur zu einer Spaltöffnung sind die bei der Berechnung der Hemmwirkung benutzten Kreise eingezeichnet.

bildung) erloschen ist, und fernerhin darin, daß diese entscheidende Teilung offenbar eine differentielle Teilung ist, sie trennt die plasmareichere Initiale von der plasmaärmeren Nachbarzelle. Der große Plasmareichtum der Initiale ist. wie in anderen Fällen solcher differentiellen Teilungen, schon unmittelbar nach der Teilung 
oder sogar schon während dieser Teilung erkennbar; es ist also ganz offensichtlich, daß hier plasmatisches Material ungleich verteilt wird und diese Inäqualität Ursache für die verschiedene Entwicklungsrichtung ist. Der Unterschied in den beiden oben genannten Möglichkeiten zur Bildung des Spaltöffnungsmusters besteht also nur darin, daß die differentielle Teilung im einen Fall an einer prädestinierten Stelle stattfindet, im anderen Fall, der uns allein beschäftigen soll, überall

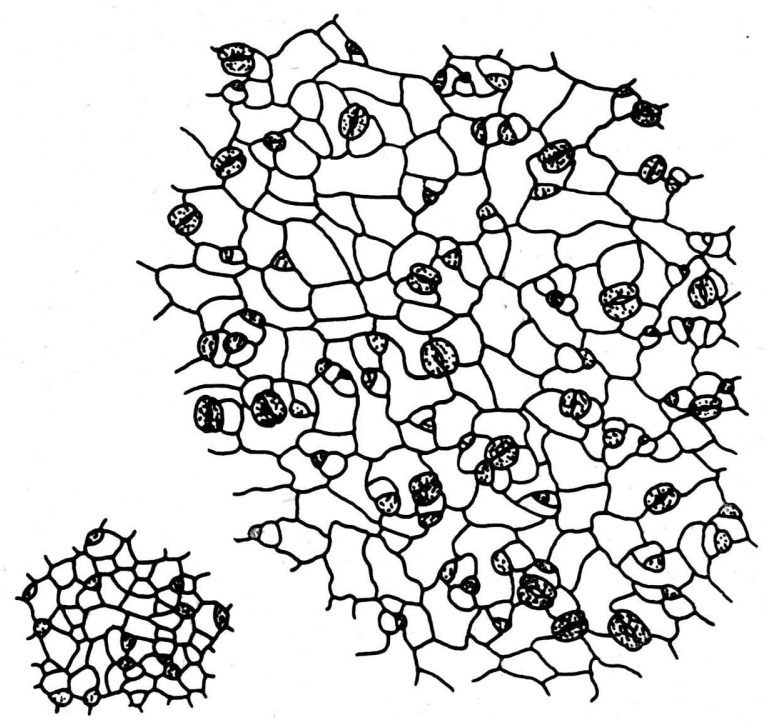

Abb. 4.

Abb. 5 .

Abb. 4. Spaltöffnungsinitialen im jungen $(0,6 \mathrm{~cm}$ breiten) Blatt von Alliaria officinalis.

Abb. 5. Neubildung von Spaltöffnungsinitialen nach der Ausweitung des ursprünglichen Spaltöffnungs(bzw. Initialen-) musters infolge des Flächenwachstums (1,5 $\mathrm{cm}$ breites Blatt von Alliaria officinalis).

möglich ist, aber nicht überall gleich häufig eintritt.

Auch beim Vorliegen dieses uns interessierenden zweiten Falles, der in der Regel (aber nicht nur) bei Dikotyledonen gegeben ist, entspricht die Verteilung derSpaltöffnungen bzw. Spaltöffnungsinitialen in der Epidermis durchaus keiner $\mathrm{Zu}$ fallsverteilung. Davon kann uns schon der bloße Anblick überzeugen. Abb. 1 zeigt uns eine Spaltöffnungsverteilung und zum Vergleich dazu Abb. 2 eine Zufallsverteilung.

Diese Zufallsverteilung wurde hergestellt, indem gewöhnliche Weizenkörner mit rotgefärbten gemischt und dann auf einem Blatt Papier ausgebreitet wurden.
Die Körner lagen in einer Ebene, aber so dicht wie möglich aneinander. Auf diesem Papier wurde dann jede Stelle, an der ein rotes Korn lag, als kleiner schwarzer Kreis eingezeichnet.

Wir können aber auch messend nachweisen, daß die Verteilung der Spaltöffnungen in der Epidermis nicht zufällig ist. Bei Sambucus nigra wurde errechnet, wie groß die Fläche ist, auf die im Durchschnitt eine Spaltöffnung entfällt. Von jedem Spaltöffnungsmittelpunkt aus wurde sodann ein Kreis mit diesem Punkt als Zentrum gezeichnet, dessen Fläche halb so groß war wie jene im Durchschnitt eine Spaltöffnung enthaltende Fläche. Ferner wurden weitere Kreise mit dem gleichen Mittelpunkt gezeichnet, deren Flächen doppelt, dreifach und vierfach so groß waren wie die jenes ersten Kreises. (In Abb. 3 sind als Beispiel nur zu einer der Spaltöffnungen diese Kreise eingezeichnet.) Jetzt lag in dem inneren Kreis natürlich je eine Spaltöffnung. Bei reiner Zufallsverteilung hätten dort noch weitere Spaltöffnungen entstehen können; die anatomischen Voraussetzungen (Zellecken) dazu waren gegeben, und in seltenen Ausnahmefällen findet man auch zwei einander so sehr benachbarte Spaltöffnungen. Da die als Ausgangspunkt gewählte Spaltöffnung schon einen gewissen Raum beansprucht, konnten in diesem Innenkreis nicht wie sonst auf einer so großen Fläche bei reiner Zufallsverteilung 0,5 weitere Spaltöffnungen erwartet werden, sondern etwas weniger als 0,5. Es trat hier aber fast nie eine zweite Spaltöffnung ein. In den sich konzentrisch anschließenden Kreisringen, die jeweils eine gleiche Fläche hatten wie der innere Kreis, waren bei rein zufallsmäßiger Verteilung durchschnittlich 0,5 Spaltöffnungen zu erwarten. Im ersten dieser Ringe, also in dem unmittelbar an den inneren Kreis anschließenden, zeigten sich aber bei Sambucus nigra nur 0,2-0,3 Spaltöffnungen. Erst im nächstfolgenden Ring ist diese Hemmung nicht mehr erkennbar, und hier ist ja auch der Raum, auf den im Durchschnitt eine Spaltöffnung entfällt, überschritten.

Natürlich variieren die Verhältnisse in quantitativer Hinsicht, aber im Prinzip besteht dieser Effekt bei der Spaltöffnungsverteilung aller untersuchten Pflanzen.

Wenn die Epidermis ein weiteres Flächenwachstum durchgemacht hat, so entfernen sich dabei natürlich die Spaltöffnungen mehr voneinander, so daß ihre neuen Abstände nicht mehr der Ausdehnung der von ihnen induzierten Hemmungsräume entsprechen. Sofern die Epidermis in diesem Zeitpunkt noch nicht zu sehr den Charakter eines Dauergewebes angenommen hat, werden jetzt zwangsläufig neue Spaltöffnungen in den Abständen eingeschoben, die der Ausdehnung jener Hemmungsräume entsprechen. Das läßt sich allerdings nur bei wenigen Pflanzen beobachten (Abb. 4,5), weil in der Regel die Entwicklungsfähigkeit zu schnell verlorengeht. 


\section{Autonomes oder abgebildetes $\mathrm{Muster}$ ?}

Nachdem festgestellt war, daß das Spaltöffnungsmuster keine Zufallsverteilung darstellt, mußte weiterhin geprüft werden, ob die spezifische Spaltöffnungsverteilung wirklich durch Wechselwirkungen zwischen den Spaltöffnungs- und gewöhnlichen Epidermiszellen selber entsteht, oder ob das Spaltöffnungsmuster vielleicht nur die $\mathrm{Ab}$ bildung eines anderen Musters im Blatt ist. Diese Möglichkeit ist grundsätzlich gegeben, und viele Muster, die im Verlaufe der Differenzierung in der Pflanze entstehen, sind so als abgebildete Muster zu begreifen.

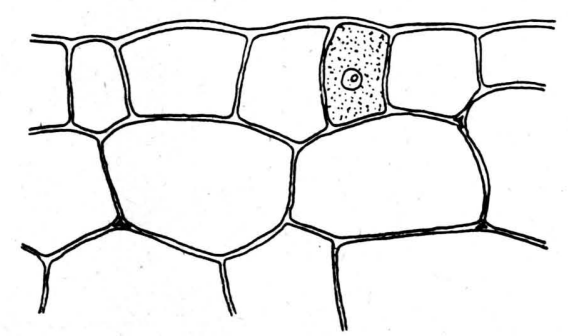

Abb. 6. Spaltöffnungsinitiale im jungen Blatt von Impatiens Sultani.

Tatsächlich ist auch im Blatt noch ein anderes Muster vorhanden, das dem der Spaltöffnungsverteilung entspricht, nämlich das aus den Atemhöhlen gebildete. Grundsätzlich wäre es denkbar, daß erst die Anlagen zu den Atemhöhlen gebildet werden und sich dann über jeder Atemhöhlenanlage eine Spaltöffnungsinitiale bildet. Natürlich müßte die Initialenbildung in diesem Falle schon dann induziert werden, wenn die Atemhöhlen noch sehr jung sind, also vielleicht nur durch einen kleinen Interzellularenraum zwischen Epidermis und subepidermaler Schicht oder innerhalb der subepidermalen Schicht angedeutet sind.

Wir haben diese Möglichkeit bei mehreren Objekten geprüft und gefunden, daß zwar schon im Zeitpunkt der Anlage der Spaltöffnungsinitialen Interzellularräume in der subepidermalen Schicht auftreten können, die Verteilung dieser Interzellularen aber keine Beziehung zur Verteilung der Spaltöffnungsinitialen hat. Außerdem zeigt Abb. 6, daß die Spaltöffnungsinitialen schon angelegt werden können, wenn sich die Epidermis noch nicht oder doch noch nicht dort, wo sich die
Initiale bildet, vom zukünftigen Mesophyll abgehoben hat. Dasselbe wurde für Asplenium nidus avis und Sambucus nigra ermittelt.

Wir dürfen also nicht das Spaltöffnungsmuster als Abbild des Interzellularenmusters auffassen, sondern umgekehrt entsteht das von den Atemhöhlen gebildete Muster dadurch, daß das stärkere Wachstum im Bereich der Spaltöffnung zwangsläufig zum Abheben der Epidermis und zur Auflockerung des Mesophylls in diesem Blatt-Teil führt. Zu dieser Schlußfolgerung kam auch schon Goebel ${ }^{1 \mathrm{a}}$.

3. Nachweis eines physiologischen Gefälles in der Hemmzone

Jede Spaltöffnungsinitiale übt auf ihre Umgebung eine Hemmwirkung aus, die, wie wir sahen, in der Nähe der Initiale am stärksten ist und sich mit etwas größerer Entfernung bald verliert. Unser Ziel war, die Grundlagen dieser Hemmwirkung ausfindig zu machen. Wir gingen dabei von der Überlegung aus, daß sich das Prinzip, das diese Hemmung ausuibt, auch in anderen Erscheinungen als in jener Hemmung äußern müsse und solche andere Erscheinungen möglicherweise Anhaltspunkte zur Ermittlung der Natur des hemmenden Prinzips selber liefern könnten.

Hinweise auf die Existenz eines physiologischen Gefälles im Umkreis einer Spaltöffnung finden sich schon in einer Arbeit $\mathrm{Pekareks}{ }^{2}$. Er fand nämlich bei Rumex acetosa ein Gefälle der Färbbarkeit mit Neutralrot. Die Schließzellen selber verhielten sich je nach den Beleuchtungsverhältnissen unterschiedlich, aber in den anschließenden Epidermiszellen bestand immer ein deutliches Gefälle in dem Sinne, daß die Färbbarkeit mit zunehmender Entfernung von den Schließzellen abnahm. Pekarek bringt dieses Gefälle damit in Zusammenhang, daß der Farbstoff nur von den Atemhöhlen her eindringen kann. Wenn diese Deutung richtig ist, dürfen wir das Gefälle der Färbbarkeit also nicht als Ausdruck eines physiologischen Gefälles innerhalb der Epidermiszellen betrachten. Allerdings sprechen Befunde von $\mathrm{R}$ e u t e $\mathrm{r}^{3}$ an der Epidermis von Polypodium vulgare doch dafür, daß hier ein physiologisches Gefälle vorliegt, denn bei diesem Objekt besteht die geringste Färbbarkeit gerade in der Nähe der Schließzellen, so daß für die Intensität der Färbung offenbar nicht die Leichtigkeit des Farbstoffzutritts entscheidend sein kann. Jedenfalls aber kann uns diese unterschiedliche Färbbarkeit noch keine näheren Aufschlüsse über die
1a K. G o e b e l, Bot. Abh. 1 [1922].
2 J. P e k a r e k, Planta 21, 419 [1933].
3 L. R e u t e r, Protoplasma 36, 321 [1942]. 
Natur des Gefälles geben, das wir hier suchen, nämlich des Gefälles in dem von den Initialen ausgehenden, die Bildung weiterer Initialen hemmenden Prinzip.

Sehr auffällig ist folgendes Gefälle: Die Zellkerne in den Epidermiszellen sind häufig in der

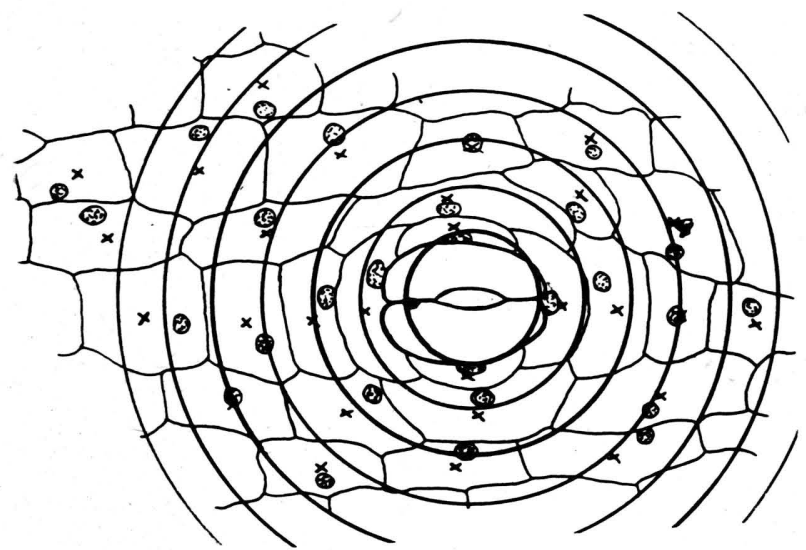

Abb. 7. Kernverlagerung in der Umgebung einer Spaltöffnung von Vriesea splendens und Einzeichnung der Kreise zur zahlenmäßigen Erfassung der Kernverlagerung.

Richtung nach den Schließzellen verlagert, und zwar um so stärker, je mehr die betreffende Epidermiszelle der Schließzelle genähert liegt. Wir haben diese nicht mehr neue Tatsache bei zahlreichen Objekten bestätigt gefunden. $K$ ü s t e $\mathrm{r}^{4}$ hat versucht, diese Orientierung der Zellkerne in den Nebenzellen als einfache Folge der besonderen Form dieser Zellen nachzuweisen. Jedoch besteht der Effekt auch noch in gewöhnlich geformten, weiter entfernt liegenden Epidermiszellen. Die positiv chemotaktische Wirkung, die die Schließzellen offenbar auf die Kerne ausüben, ist um so schwächer, je weiter die betr. Epidermiszellen von den Schließzellen entfernt liegen. Das zeigt eine genauere zahlenmäßige Erfassung, die wir für Vriesea splendens durchgeführt haben. In den Zeichnungen von vier solchen Spaltöffnungen wurden, mit dem Spaltöffnungsmittelpunkt als Zentrum, konzentrische Kreise gezeichnet, von denen der Radius des innersten so gewählt wurde, daß er die seitlichen Außenwände der Schließzellen, oder wenigstens eine von ihnen, berührte (Abb. 7); die nächsten Kreise folgten mit Radien, die jeweils um den gleichen Betrag (dessen relative Größe aus Abb. 7 ersichtlich ist) größer waren. Sodann wurde der (nach Augenmaß geschätzte) Mittelpunkt jeder Epidermiszelle durch

${ }^{4}$ E. K ü ste r, Flora 97, 1 [1907]. ein Kreuz markiert und fernerhin geprüft, ob die Kerne, die in die einzelnen konzentrischen Kreise fielen, jeweils von diesem Mittelpunkt aus nach innen oder nach außen verlagert waren; im ersteren Fall, also bei Verlagerung zu den Schließzellen hin, wurde von ,genäherten Kernen“ gesprochen. Folgende Tabelle bringt die Ergebnisse als Mittelwerte aus den vier Messungen:

\begin{tabular}{|c|c|}
\hline $\begin{array}{c}\text { Bezeichnung der Kreis- } \\
\text { ringe, in der Reihenfolge, } \\
\text { wie sie dem Innenkreis } \\
\text { folgen }\end{array}$ & $\begin{array}{c}\text { Prozentsatz genäherter } \\
\text { Kerne unter den auf diesen } \\
\text { Kreisring entfallenden } \\
\text { Kernen }\end{array}$ \\
\hline $\begin{array}{l}\text { 1. } \text { Kreisring } \\
2 . \quad " \\
\text { 3. } \quad " \\
\text { 4. } " \\
\text { 5. } "\end{array}$ & $\begin{array}{r}100 \% \\
86 \% \\
84 \% \\
42 \% \\
50 \%\end{array}$ \\
\hline
\end{tabular}

Für den ersten, zweiten und dritten Kreisring läßt sich also eine nach außen schwächer werdende Anziehung der Zellkerne durch die Spaltöffnung feststellen.

In Abb. 8 sind vier Spaltöffnungen von Vriesea splendens so gezeichnet, wie sie innerhalb der
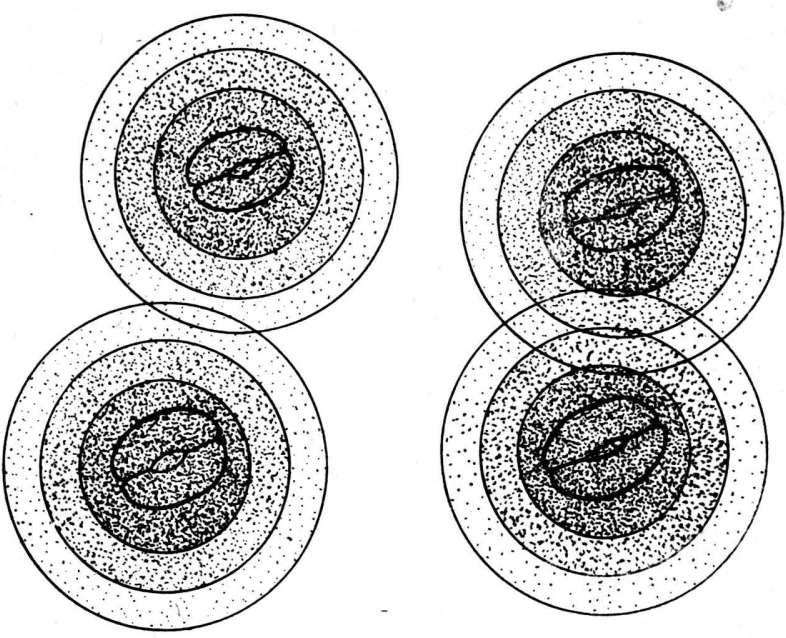

Abb. 8. Vier Spaltöffnungen aus dem Blatt von Vriesea splendens und Einzeichnung der Kreisflächen, in denen eine Kernverlagerung feststellbar ist.

Epidermis verteilt waren. Außerdem wurden um jede Spaltöffnung die oben genannten drei Kreisringe gezeichnet, in denen die Kernverlagerung nach innen nachweisbar war. Die verschiedene Dichte der Punktierung soll die unterschiedliche Stärke der chemotaktischen Anziehung kennzeichnen. Man sieht, daß die Zone, in der eine 
Anziehung nachweisbar ist, nicht viel kleiner ist als der Flächenbereich jener Hemmwirkung, also als die Fläche, in der eine Spaltöffnung nicht die Bildung einer zweiten zuläßt. Die Kernverlagerung ist also ein ziemlich empfindlicher "Test" für das Vorhandensein des gesuchten chemischen Gefälles, das der Hemmwirkung zugrunde liegen muß.

Gegen diese Konsequenz könnte man noch Bedenken haben, wenn man annimmt, diese Kernverlagerung habe nichts mit der Wirkung des Gefälles zu tun, das der Hemmung zugrunde liegt, sondern sei Folge eines erst später, nach der Fertigstellung des Spaltöffnungsmusters auftretenden Gefälles. Ein solches späteres Gefälle könnte man vermuten, weil die Schließzellen eine Assimilationstätigkeit zeigen und infolgedessen sehr wohl durch die Anziehung von Stoffen, die bei der Assimilation verbraucht werden oder durch die Abgabe von Stoffen, die bei der Assimilation entstehen, sekundär ein stoffliches Gefälle in den Nachbarzellen verursacht werden könnte.

Um diese Möglichkeit auszuschließen, prüften wir noch die Kernverlagerung in ganz jungen Epidermiszellen, in einem Stadium, das erst junge, noch nicht assimilierende Schließzellen oder überhaupt erst die Initialen vor ihrer Aufteilung in die späteren Schließzellen aufwies. Die Abb. 9, ebenso wie alle übrigen aus einem größeren Material ausgewählt, zeigt, daß sich die Kernverlagerung auch schon in sehr jungen Entwicklungsstadien nachweisen läßt, jener mögliche Einwand also entfällt. Daß die Kernverlagerung nicht Folge der spezifischen Tätigkeit der Schließzellen ist, ergibt sich außerdem noch aus einer anderen Beobachtung: Die Kernverlagerung zeigt sich nicht nur im Umkreis der Spaltöffnungsinitialen und Spaltöffnungen, sondern auch im Umkreis von Haarinitialen und Haaren bzw. Drüsen (Abb.10). Bei der Gelegenheit verdient es Erwähnung, daß sich solche Emergenzen durchaus in das von den Spaltöffnungen gebildete Muster einfügen können (Abb. 11). D. h. die Hemmzone um eine Spaltöffnungsinitiale verhindert auch die Entstehung eines Haares, und dieses selber bildet um sich eine Hemmzone, die nicht nur die Entstehung weiterer Haare, sondern auch die Entstehung von Spaltöffnungen unterdrückt. Die von Haaren, namentlich von großen Haaren, Drüsenschuppen usw. gebildete Hemmzone ist meist größer als die von den Spaltöffnungen gebildete.
Man darf also wohl annehmen, daßs Spaltöffnungen, Haare usw. von zunächst gleichwertigen Initialen ausgehen, daß diese Initialen aber ähnlich wie die embryonalen Epidermiszellen nochmals einen „Kippvorgang" durchmachen können, der aus ihnen entweder Spaltöffnungen oder jene Emergenzen entstehen läßt. $\mathrm{Zu}$ den Faktoren, die diesen Kippvorgang im Sinne der Haarbildung begünstigen können, gehört offenbar die Gegenwart von Leitbündeln, über denen keine Spaltöffnungen, oft aber statt dessen Haare oder Drüsenschuppen stehen (Abb. 11).

\section{Die chemische Natur der Hemm- wirkung}

In der Kernverlagerung haben wir einen Test für das Vorhandensein und die Stärke des Hemmfeldes um eine Spaltöffnung gefunden. Um die Natur des hemmenden Prinzips selber ausfindig zu machen, fragten wir nun zunächst, welche Faktoren sonst eine positive Chemotaxis der Kerne verursachen können.

Eine Kernverlagerung läßt sich bekanntlich leicht durch Verwundung bzw. durch Wundhormone erzielen ${ }^{5}$. Aus dieser Tatsache schienen uns für die Frage nach den Ursachen der Kernverlagerung im Umkreis einer Spaltöffnung zwei Möglichkeiten zu folgen: die traumatisch bedingte Kernverlagerung könnte entweder durch eine erhöhte Acidität bedingt sein (Aciditätserhöhung als Wundwirkung ist bekannt), oder es könnten Teilungshormone von der Art der Wundhormone verantwortlich sein.

Zunächst haben wir die erste. Möglichkeit geprüft. Sie schien uns schon aus folgendem Grunde diskutabel zu sein: im Laubblatt ist, jedenfalls bei einigen daraufhin untersuchten Objekten ${ }^{6}$, die Acidität der Unterseite niedrigêr als die Acidität der Oberseite. Dieser Unterschied könnte möglicherweise auch dafür verantwortlich sein, daß sich auf der Unterseite mehr Spaltöffnungen bilden als auf der Oberseite; wenn sich dann auf der Unterseite um jede Spaltöffnungsinitiale ein Hof größerer Acidität ausbildet, so wäre dieser als Ursache für die Ausschließung der Bildung weiterer Spaltöffnungsinitialen anzusehen.

Wenn diese Möglichkeit den Tatsachen entspricht, so sollte man erwarten, daß durch experimentelle Erhöhung der Acidität eine Verminderung der Spaltöffnungsdichte, durch experimentelle Erniedrigung der Acidität eine Erhöhung

5 W. L o o s, Protoplasma 14, 331 [1931].

${ }^{6}$ E. B ünn i n g, Z. Bot. 37, 433 [1942]. 
der Spaltöffnungsdichte eintritt. Entsprechende Versuche haben wir durch Behandlung junger Blattanlagen, deren Epidermis gerade vor dem Stadium der Spaltöffnungsinitialen-Bildung oder

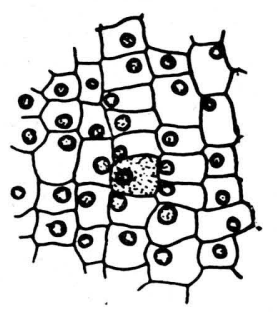

Abb. 9 .

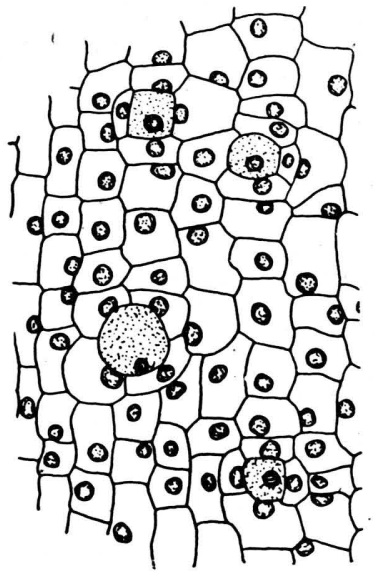

Abb. 10.
Abb. 9. Kernverlagerung in der Nähe einer Spaltöffnungsinitiale von Karatas Carolina.

Abb. 10. Kernverlagerung in der Nähe von zwei noch ungeteilten Initialen für Spaltöffnungen (links unten und rechts oben) und zwei ebenfalls noch ungeteilten

Initialen für Saugschuppen bei Karatas Carolina.

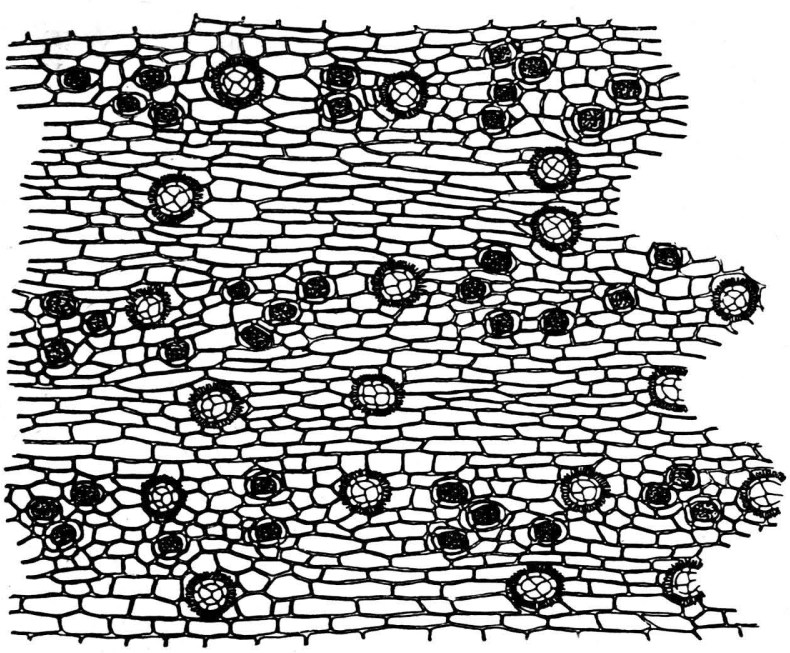

Abb. 11. Vriesea hieroglyphica. Verteilung der Spaltöffnungen und Saugschuppen in der Epidermis.

in diesem Stadium stand, unternommen, indem die Blattanlagen zur Hälfte mit sauren bzw. alkalischen Lösungen bestrichen wurden, während die andere Hälfte jedes so behandelten Blattes zur Kontrolle diente. Der Erfolg dieser Behandlung lag wohl im Sinne der Erwartung, war aber nicht immer sehr stark (Abb. 12,13). Auch glichen sich die Unterschiede mit länger dauernder Entwicklung oft wieder aus. Aus diesem Grunde sowie auch deshalb, weil selbst mit den am stärksten sauren bzw. alkalischen, von den Blättern noch

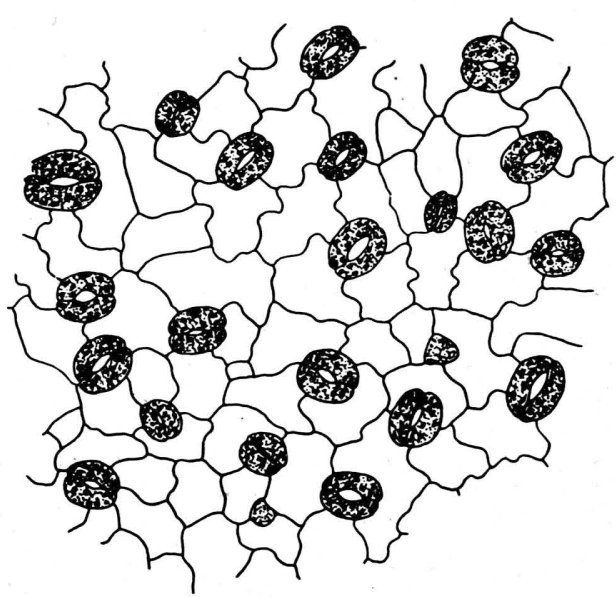

Abb. 12 a. Verteilung der Spaltöffnungen im Blatt von Impatiens Sultani, 10 Tage nachdem die Blattanlage im Stadium der Spaltöffnungsbildung mit $n / 100-\mathrm{KOH}$ bestrichen worden war. Die alkalische Lösung fördert die Spaltöffnungsbildung.

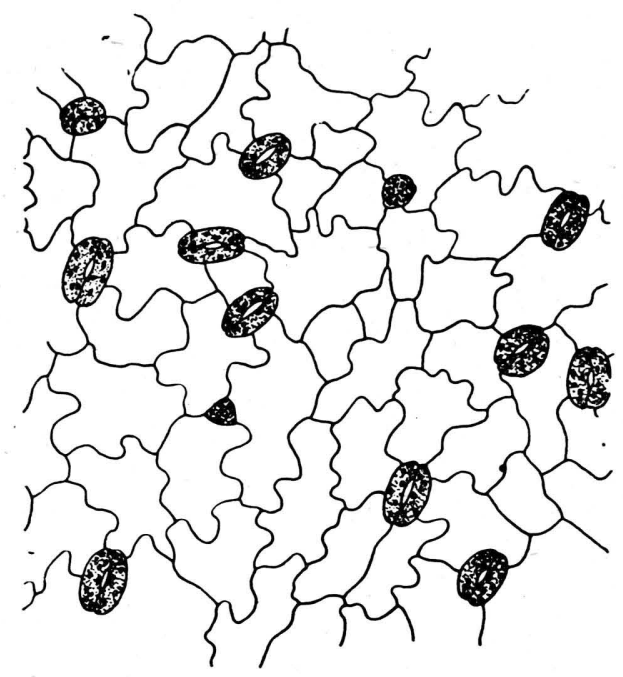

Abb. 12 b. Kontrolle (nicht behandelte Hälfte des gleichen Blattes).

eben ertragenen Lösungen nie eine völlige Unterdrückung bzw. eine übermäßig starke Steigerung der Spaltöffnungsbildung zu erzielen war, schien es uns recht zweifelhaft, ob man berechtigt ist, die Hemmung, die eine Spaltöffnungsinitiale auf ihre Umgebung ausübt, als Säurewirkung zu betrachten. 
Diese Zweifel werden noch verstärkt, wenn man beachtet, daß wohl die Schließzellen selber eine erheblich andere Acidität haben als die übrigen Epidermiszellen, aber in den an die Schließzellen anschließenden Epidermiszellen zum mindesten kein leicht nachweisbares, also jedenfalls kein starkes Gefälle

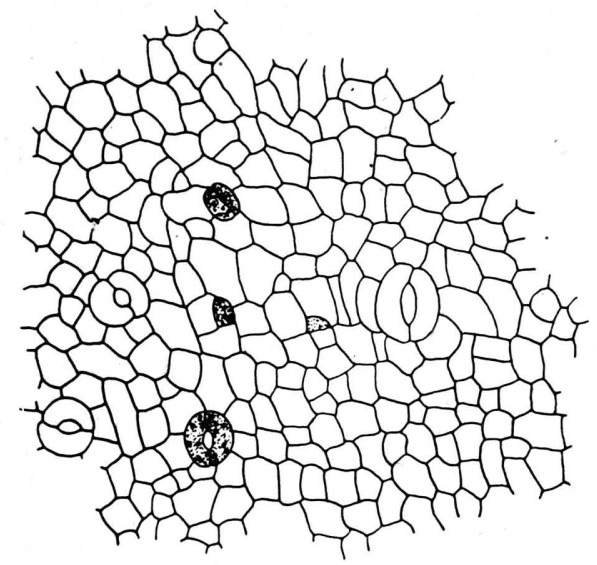

Abb. 13 a. Wie Abb. 12, jedoch 10 Tage mit $n / 15-\mathrm{KH}_{2} \mathrm{PO}_{4}$ behandelt. Die saure Lösung hemmt die Spaltöffnungsbildung. Die ohne Inhalt gezeichneten Schließzellen sind tot.

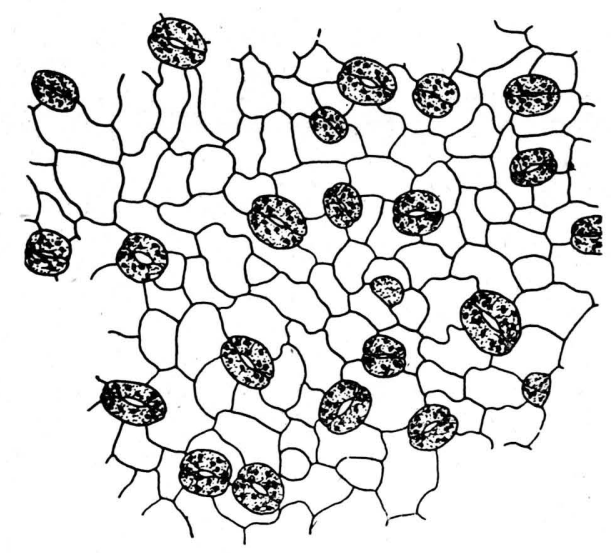

Abb. 13b. Kontrolle (nicht behandelte Hälfte des gleichen Blattes).

der Acidität besteht. Außerdem kann die Acidität der Schließzellen bei einigen Objekten höher, bei anderen niedriger sein als die der Nebenzellen (vgl. P e k a$\mathrm{rek}^{2}, \mathrm{R}$ e u te $\mathrm{r}^{3}$ ).

Wir haben in diesem Zusammenhang übrigens auch noch Versuche über die Einwirkung von $\beta$ Indolylessigsäure (in Pastenform) unternommen und gefunden, daß hierbei eine ähnliche Beeinflussung eintritt wie durch die Säure, nämlich geförderte Zellteilung und gehemmte Spaltöffnungsbildung (Abb.14); erst wenn diese geförderten
Teilungen beendet sind, tritt eine, dann besonders lebhafte Initialenbildung ein.

Der geringe Erfolg der Versuche über die Wirkung von Säuren und Laugen läßt uns vermuten, daß doch vielleicht die andere der beiden oben ge-

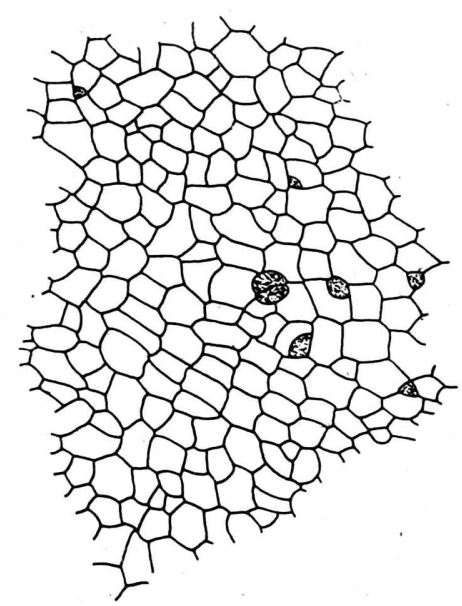

Abb. 14 a. Wie Abb. 12, jedoch 14 Tage mit Wuchsstoffpaste behandelt.

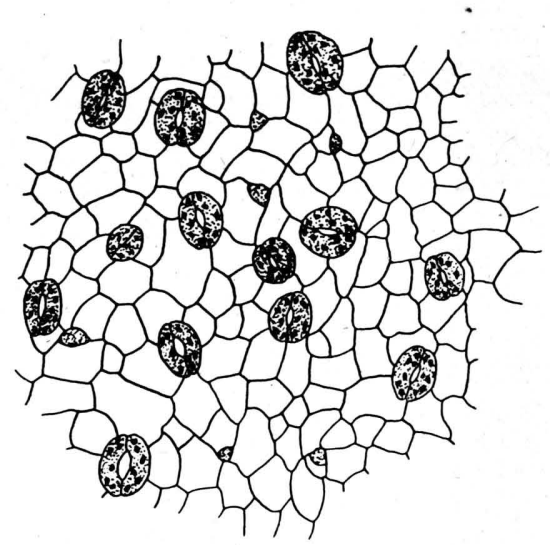

Abb. 14 b. Kontrolle (nicht behandelte Hälfte des gleichen Blattes).

nannten Möglichkeiten für die Ursachen der Kernverlagerung der Lösung unseres Problems näherführt, obwohl der Hinweis, daß Teilungsförderung die Initialenbildung unterdrückt, wertvoll ist. Wir gingen jetzt also von der Annahme aus, daß eine Verwundung durch die Vermittlung spezifischer Stoffe, d.h. etwa durch Vermittlung des Wundhormons, zur Kernverlagerung in den Nachbarzellen führt; diese Vermutung wird auch durch die Arbeit von L o o s ${ }^{5}$ erheblich gestützt.

Natürlich kann die Bildung der Spaltöffnungs- 
initiale in keiner Weise mit einer Verwundung verglichen werden; aber damit wird nicht die Möglichkeit ausgeschlossen, daß auch bei einem so normalphysiologischen Prozeß wie der Differenzierung von Spaltöffnungen Stoffe wirksam werden, die sonst als Wundhormone auftreten.

Für diese Möglichkeit sprach schon eine andere bekannte Tatsache: In der Nähe einer Spaltöffnungsinitiale treten oft neue Zellteilungen auf, die zur Bildung von „Nebenzellen“ führen. Man darf diese neuen Teilungen in den Nachbarzellen durchaus nicht als gleichwertig mit den Teilungen in der Initiale betrachten und die Weiterentwicklung der Initiale

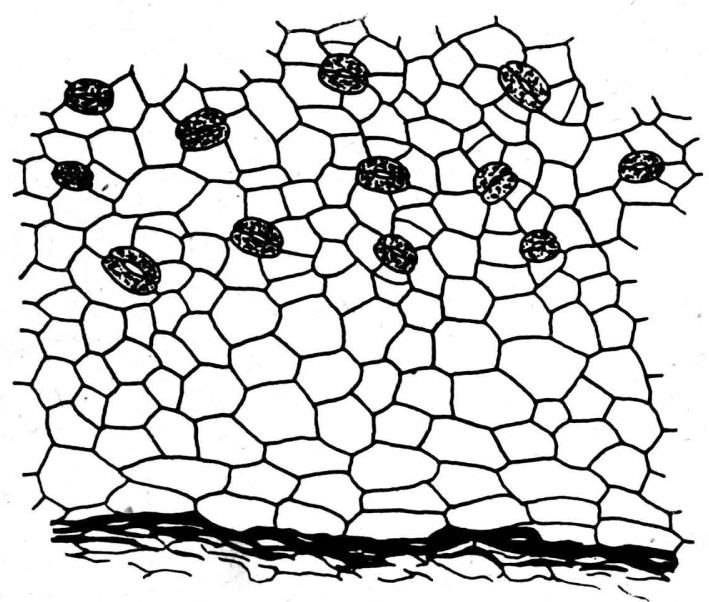

Abb. 15. Unterdrückung der Spaltöffnungsbildung in der Nähe einer Schnittwunde im Blatt von Theobroma Cacao.

gemeinsam mit den erneuten Teilungen in der Umgebung nur als Folge des Wiederembryonalwerdens eines größeren Zellkomplexes auffassen, der Initiale und Nachbarschaft umfaßt, und in dem die Initiale eben nur noch etwas mehr embryonal ist als ihre Umgebung. Diese Deutung ist unrichtig, weil sich die Initiale und die neu zur Teilung schreitenden Nachbarzellen in ihren Leistungen grundsätzlich unterscheiden: aus jener wird eine Spaltöffnung und in diesen ist die Spaltöffnungsbildung geradezu unterdrückt. Auch die anatomische Untersuchung zeigt ja, daß sich zunächst nur die Initialen scharf von ihrer Umgebung abheben, und wo diese Initialen entstanden sind, wird erst sekundär - also offenbar bedingt durch die Initiale, nicht bedingt durch den Faktor, der die Initialenbildung verursachte - die Teilungstätigkeit in den Nachbarzellen wieder in Gang gesetzt. Die Annahme, daß die Initiale ein Hormon an die Umgebung abgibt, das wir in seiner Wirkung mit dem Wundhormon vergleichen dürfen, ist also durchaus naheliegend.

Zur Prüfung unserer Vermutung wurde untersucht, wie eine experimentelle Anreicherung mit
Wundhormon auf die Bildung von Spaltöffnungen wirkt. Dazu haben wir einmal junge Blätter, in denen die Spaltöffnungsbildung noch nicht begonnen hatte, durch leichte Einschnitte verletzt und nach längerer Zeit wieder untersucht. Es zeigte sich dann (bei Theobroma Cacao und Impatiens Sultani), daß in der Nähe der Wunde -

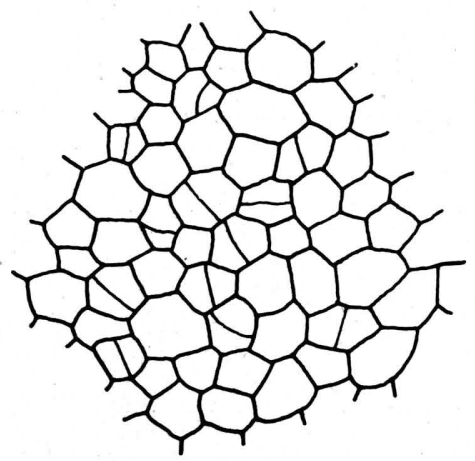

Abb. 16a. Unterdrückung der Spaltöffnungsbildung bei Theobroma Cacao infolge des Bestreichens mit Gewebebrei aus jungen Bohnenhülsen im Stadium der Spaltöffnungsbildung, gezeichnet 10 Tage nach Beginn der Behandlung.

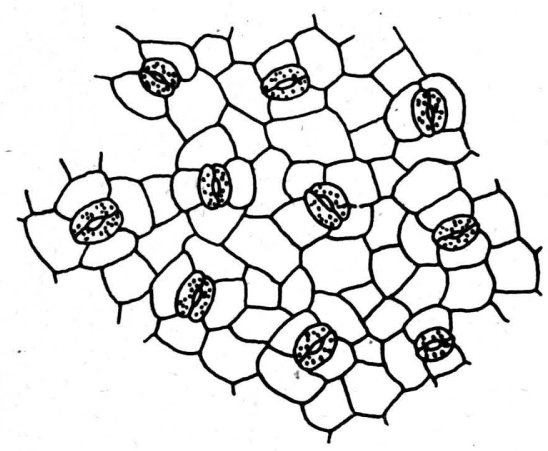

Abb. 16b. Unbehandelte Stelle aus dem gleichen Blatt.

wie zu erwarten war - neue Zellteilungen eingetreten waren, außerdem aber war die Bildung von Spaltöffnungen tatsächlich bis auf eine ziemlich weite Entfernung hin unterdrückt, und zwar erheblich weiter, als die Förderung der Zellteilungen zu beobachten war (Abb. 15). Das harmoniert durchaus mit den Beobachtungen am Spaltöffnungsmuster, auch hier sind ja die zur Nebenzellbildung führenden geförderten Zellteilungen auf eine viel kleinere Zone beschränkt als die Unterdrückung der Bildung weiterer Spaltöffnungsinitialen.

Weiterhin haben wir jungen Blättern der gleichen Objekte, wieder im Stadium der beginnenden 
Anlage von Spaltöffnungsinitialen, nicht durch Verwundungen, sondern durch Auftragen von Gewebebrei aus Hülsen junger Bohnen das Wundhormon experimentell zugeführt. Das Resultat war gleichartig: Stellenweise neue Zellteilungen, aber auch dort, wo diese nicht aufgetreten waren, gehemmte Spaltöffnungsbildung (Abb. 16).

Wir kommen also zu dem Schluß, daß das regelmäßige Spaltöffnungsmuster offenbar entsteht, indem dort, wo ,zufällig“ eine Initiale aufgetreten ist, diese eine teilungsfördernde Substanz an die Umgebung abgibt und dadurch die Bil-

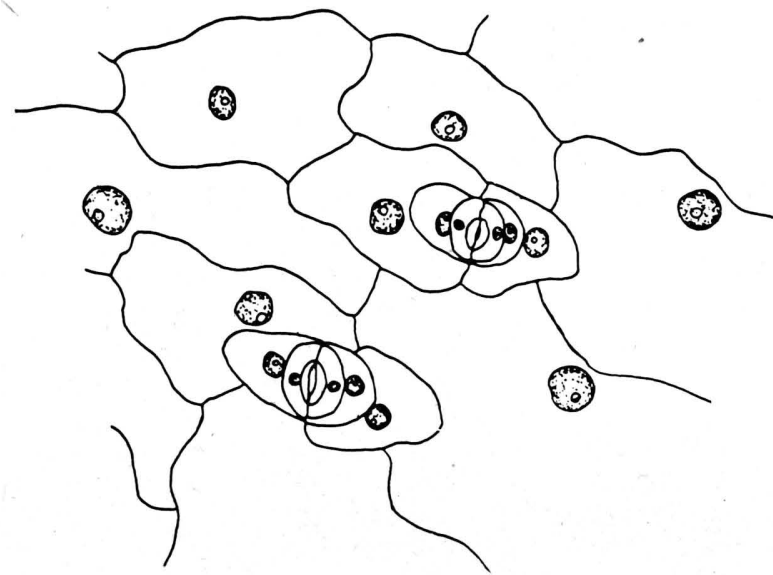

Abb. 17. Portulaca grandiflora. Unterschiedliche Größe der Kerne in den Spaltöffnungsnebenzellen und in den gewöhnlichen Epidermiszellen.

\section{dung weiterer Spaltöffinungsinitialen unterdrückt wird.}

Fassen wir noch einmal die Gründe für diese Konsequenz zusammen. Ein Teilungshormon (Wundhormon) kann offensichtlich folgende Effekte ebenso wie eine Spaltöffnungsinitiale hervorrufen: Erneuerung der Teilungstätigkeit (nur in ausreichend starker Konzentration des Hormons), Hemmung der Spaltöffnungsbildung (auch in geringerer Konzentration) und positiv chemotaktische Bewegung von Zellkernen.

Hier sei noch auf eine Tatsache hingewiesen, die bereits bekannt ist und bei der weiteren Analyse der besprochenen Vorgänge wichtig werden könnte. Die Kerne in den Nebenzellen sind oft erheblich kleiner als in den gewöhnlichen Epidermiszellen (Abb. 17, vgl. auch $\mathrm{K}$ ü s te $\left.\mathrm{r}^{4}\right)$. Wir wissen nun durch zahlreiche neuere Erfahrungen (vgl. z. B. Geitle ${ }^{7}$ ), daß die Kerne im somatischen Gewebe der Pflanzen

7 L. Ge i t l e r, Ber. dtsch. bot. Ges. 58, 131 [1940].

8 E. Strasburger, Jb. wiss. Bot. 5, 297 [1866]. oft einen hohen Polyploidiegrad aufweisen. Nach den Erfahrungen, die hierüber vorliegen, könnte man annehmen, daß auch die Kerne der gewöhnlichen Epidermiszellen infolge dieser somatischen Polyploidie so stark gewachsen sind, während in den Nebenzellen vielleicht infolge der Gegenwart des von den Schließzellen abgegebenen Teilungshormons die Spindelbildung und so die Teilung ermöglicht und dadurch das extreme Anwachsen der Kerne verhindert wird.

\section{Die Zellteilungen bei der Initialenbildung}

Nachdem wir die Bildung des Spaltöffnungsmusters untersucht haben, wollen wir jetzt noch den Vorgang der Bildung der einzelnen Initiale etwas näher betrachten. Daß es sich bei der Initialenbildung regelmäßig um die Abtrennung einer plasmareichen kleinen Zelle von einer benachbarten größeren plasmaarmen handelt, ist schon durch eingehende Untersuchungen S t r a s burgers ${ }^{8}$ an zahlreichen Objekten bekannt geworden.

Leider lassen die meisten Objekte keine genauere Untersuchung $\mathrm{zu}$, da die Zellen bei der Initialenbildung sehr klein sind. Ein günstiges Material stellen Begonia-Arten dar. Namentlich B. heracleifolia haben wir näher untersucht. Bei dieser Art finden noch dann, wenn das Blatt bereits 1-2 cm lang ist, regelmäßig Initialenbildungen statt; die Epidermiszellen sind dann schon erheblich größer als bei den meisten anderen Pflanzen im Stadium der Bildung von Spaltöffnungsinitialen. Diese Epidermiszellen sind jetzt auch schon stark vakuolisiert, das Plasma findet sich nur noch in Form eines wandständigen Plasmaschlauches und einiger den Saftraum durchziehender Stränge.

Die weiteren Teilungen in diesen vakuolisierten Epidermiszellen finden so statt, wie es S in not und $\mathrm{B} l$ o $\mathrm{ch}^{9}$ für die vakuolisierten Zellen anderer Pflanzen beschrieben haben: Der wandständige Kern wird ins Zellinnere gezogen und während der Prophase bildet sich eine Plasmalamelle, ein sog. Phragmosom, aus dem später die neue Wand hervorgeht.

Die Bildung der Spaltöffnungsinitiale bei Begonia heracleifolia beginnt damit, daß eine 5-6eckige Epidermiszelle durch eine Wand halbiert wird. Wir sehen in der noch ungeteilten Epidermiszelle den Kern in die Mitte rücken; noch bevor er sich teilt, wird das erwähnte Phragmosom angelegt. Sowohl am lebenden Material als auch an dem mit Alkohol-Eisessig fixierten und mit Karminessigsäure gefärbten läßt sich dieses Phragmosom leicht erkennen. Nach der Teilung sammelt sich in einer der beiden Tochterzellen

${ }^{9}$ E. W. Sinnot u. R. Bloch, Amer. J. Bot. 18,225 [1941]. 
das Plasma in der Mitte der neuen Wand; auch der Zellkern bleibt an dieser Stelle (Abb. 18). Das so angehäufte Plasma trennt sich nun durch zwei weitere Wände, die ebenfalls als Phragmosom beginnen, von der plasmaärmeren Umgebung, so daß die fertige Initiale entsteht, aus der sich durch eine nochmalige Teilung die beiden Schließzellen bilden.

Der ganze Vorgang bedarf noch einer genaueren Untersuchung. Jedoch handelt es sich hierbei um ein nicht für die Physiologie der Spaltöffnungsbildung spezifisches Problem. Auch andere inäquale Teilungen, z. B. die zur Bildung von Wurzelhaarinitialen
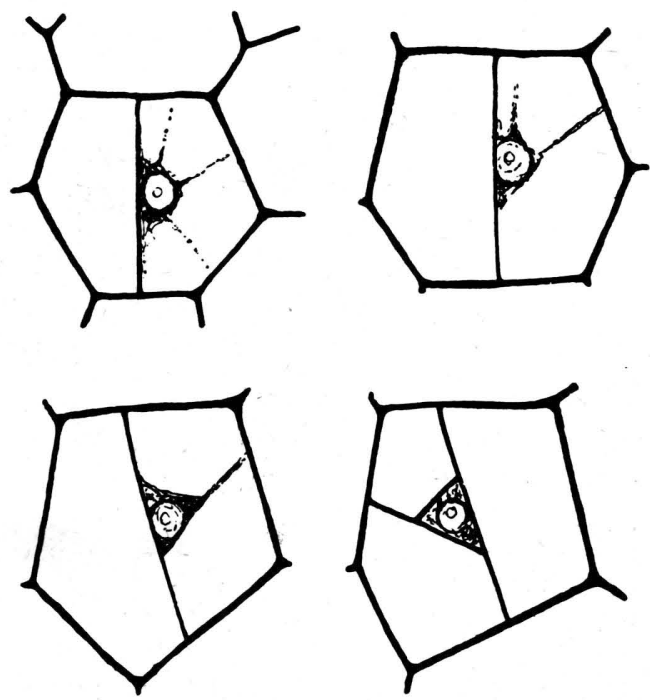

Abb. 18. Bildung einer Spaltöffnungsinitiale bei Begonia heracleifolia.

führenden, beginnen mit einer polaren Plasmaansammlung, der die Teilung erst folgt. Dieses Problem der inäqualen Teilung soll daher in einem allgemeineren Zusammenhang untersucht werden.

\section{Die Bildung von Spaltöffnungs -} gruppen

Bei einigen Pflanzen, zu denen namentlich mehrere Begonia-Arten gehören, sind die Spaltöffnungen gruppenweise in dẹr Epidermis angeordnet. Jede Gruppe kann etwa 2-5 sehr nahe beieinander liegende Spaltöffnungen umfassen; die Gruppen sind voneinander ebensoweit entfernt, wie bei anderen Pflanzen die einzelnen Spaltöffnungen voneinander getrennt sind (Abb. 19). Diese Verteilung ist nach der bisher gewonnenen Vorstellung nicht ohne weiteres verständlich.

Die Initialen, die eine Gruppe von Spaltöffnungen entstehen lassen, bilden sich nicht gleichzei- tig, sondern deutlich nacheinander. Die Bildung der ersten Initiale im Blatt von Begonia heracleifolia haben wir schon beschrieben. Die Ent-

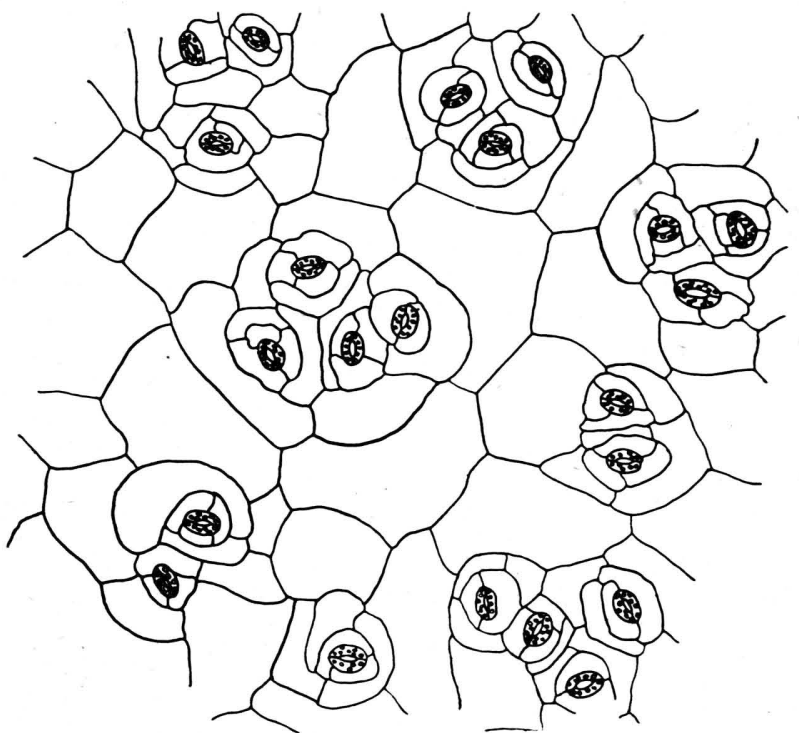

Abb. 19. Spaltöffnungsgruppen im Blatt von Begonia semperflorens.
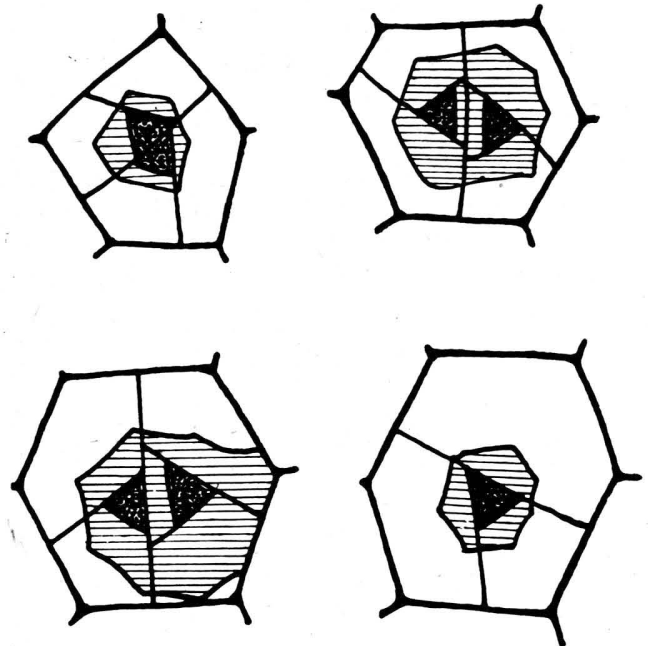

Abb. 20. Bildung von Spaltöffnungsinitialen bei Begonia heracleifolia. Der Interzellularraum zwischen Epidermis und Mesophyll ist schraffiert.

stehungsweise der weiteren Initialen ergibt sich aus Abb. 20.

Warum übt nun hier die erstgebildete Initiale nicht die vorher beschriebene Hemmwirkung aus, während die ganże Gruppe offenbar doch die gleiche Hemmung bewirkt, die sonst von der einzelnen Spaltöffnungsinitiale ausgeht? Wir können nur auf die Beobachtung hinweisen, daß sich 
unterhalb der ersten Initiale unmittelbar nach ihrer Entstehung ein Interzellularraum bildet, der die Initiale vom Mesophyll trennt (Abb. 20). Dieser Interzellularraum wird rasch größer. Das Gebiet, in dem weitere Initialen entstehen können, deckt sich mit der Ausdehnung dieses Interzellularraums, und nie bildet sich eine neue Initiale, wenn die betr. Stelle der Epidermis nicht schon durch den Interzellularraum vom Mesophyll getrennt ist. Nur die Bildung der ersten Initiale ist - wie bei anderen Pflanzen - nicht an das Vorhandensein einer Interzellulare gebunden. Offenbar hebt also der Interzellularraum im Bereich seiner Ausdehnung die von der ersten Initiale ausgehende Hemmwirkung auf, und nur außerhalb seines Bereichs kann die Hemmwirkung wieder zur Geltung kommen.

Natürlich braucht man nicht anzunehmen, daß die Gegenwart von Luft auf der Innenseite der Epidermis das hemmende bzw. positive, Chemotaxis auslösende Prinzip direkt inaktiviert, vielmehr dürfte es sich eher so verhalten, daß zur Entfaltung jener von den Initialen ausgehenden Wirkungen auch stoffliche Einflüsse vom Mesophyll notwendig sind, die beim Vorhandensein von Interzellularen ausgeschaltet werden.

7. Diskussion der Ergebnisse und Hinweise auf die Bildung anderer $\mathrm{Muster}$

Die Anzahl der Teilungen, die in einer jungen Blattanlage noch stattfinden, ist sehr beschränkt. Wir wissen, daß bei der Entwicklung der pflanzlichen Gewebe die Zellen nach ihrer Abtrennung vom eigentlichen Meristem nur noch eine geringe Anzahl von Teilungen, oft z. B. 2-4 Teilungen, durchführen. Dann hört die Teilungstätigkeit vollständig auf, und die weitere Differenzierung beginnt.

Offenbar bekommen also die vom Meristem abgetrennten Zellen noch einen gewissen Betrag eines für die Teilungstätigkeit wichtigen Stoffes, etwa eines Teilungshormons, mit; ist diese Mitgift verbraucht, so wird die Teilungstätigkeit beendet.

Eine weiterhin anscheinend häufige Erscheinung bei der pflanzlichen Gewebebildung ist es, daß dann, wenn die Teilungstätigkeit annähernd erloschen ist, also jenes „Teilungshormon“ fast verbraucht wurde, differentielle Teilungen auftreten, die dann - je nach dem Gewebe, um das es sich handelt - zur Abschnürung von Initialen führen, aus denen etwa Wurzelhaare, Idioblasten

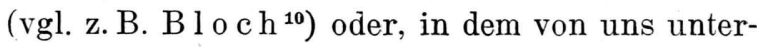
suchten Fall, Spaltöffnungen werden. Bei diesen differentiellen Teilungen entsteht immer eine sehr plasmareiche Zelle, die zu jener Initiale wird, und eine plasmaärmere. Warum diese differentielle Teilung erst während des Erlöschens der Teilungstätigkeit eintreten kann, vermögen wir noch nicht zu sagen.

Die differentielle Teilung schafft völlig neue Bedingungen. Die plasmareiche Initiale hat viele Eigenschaften der embryonalen Zellen wieder gewonnen; sie kann (etwa bei der Bildung von Idioblasten ${ }^{10}$ ) kompliziertere Wachstumsleistungen als die Schwesterzelle durchführen, sie kann oft aber auch ein Wiederaufleben der Teilungstätigkeit zeigen.

So trennt z.B die differentielle Teilung bei der Spor-Elater-Teilung der Lebermoose eine Zelle, die sich nicht mehr teilt, von der anderen, plasmareicheren ab, die mehrere Teilungen durchführt und so die Sporen bildet. Bei Chara führt die differentielle Teilung in den von der Scheitelzelle abgeschnürten Segmenten ebenfalls zur Bildung einer plasmareichen Zelle, die zahlreiche Teilungen durchführen kann, und einer plasmaärmeren, die ungeteilt bleibt und zur Internodialzelle wird. Im jungen Phloem trennt eine differentielle Teilung die zukünftige Siebröhre von einer plasmareicheren Nachbarzelle, in der allein noch weitere Teilungen stattfinden können, die zur Bildung der Geleitzellen führen. Diese Beispiele lieBen sich durch zahlreiche andere ergänzen.

Die Anzahl von Teilungen, die eine solche durch differentielle Teilung entstandene Initiale noch durchzuführen vermag, ist oft, wie schon die obigen Beispiele zeigen, beschränkt. In dem von uns untersuchten Fall, also in der Schließzelleninitiale, tritt nur noch eine Teilung ein. Wird aus der Initiale ein Haar, eine Drüsenschuppe usw., so ist die Anzahl von Teilungen größer.

Diese Initialen in der Epidermis, mögen es nun Initialen sein, die zu Spaltöffnungen werden, oder solche, die Emergenzen entstehen lassen, nehmen ihre besondere Entwicklungsrichtung nicht schon einfach darum ein, weil ihnen durch die differentielle Teilung wieder die Teilungsfähigkeit gegeben ist, sondern es sind noch andere Eigenschaften wichtig, die sie gleichzeitig mit der differentiellen Teilung erworben haben; denn die Teilungsfähigkeit allein genügt, wie die fehlende Spaltöffnungs- und Haarbildung in der ganz jungen Epidermis oder im Bereich der sich lebhaft teilenden Spaltöffnungsnachbarzellen

10 R. B l o ch, Amer. J. Bot. 33, 544 [1946]. 
zeigt, durchaus nicht, um in einem bestimmten Alter eine Spaltöffnung oder ein Haar entstehen zu lassen.

Eine, aber auch nur eine der Fähigkeiten, die die Initialen für Spaltöffnungen und Emergenzen mit der differentiellen Teilung erwerben, wirkt sich auch auf die Nachbarschaft dieser Initialen aus; die Initialen geben nämlich von ihren Teilungsstoffen an die Umgebung ab. Diese Anwesenheit von Teilungsstoffen allein, ohne die den Initialen zukommenden weiteren Fähigkeiten (die irgendwie an die Plasmaanhäufung gebunden sein müssen), ist, wie schon die fehlende Spaltöffnungsbildung in der noch zu jungen Epidermis lehrt, ein Hindernis für das Eintreten der differentiellen Teilung, die zur Bildung der Initialen notwendig ist. So wird uns die Tatsache, daß Teilungsstoffe (die offenbar mit dem Wundhormon identisch sind, zum mindesten so wirken wie dieses) eine Spaltöffnungsbildung im Umkreis einer Initiale unterdrücken, zwar durchaus noch nicht verständlich, aber diese Tatsache harmoniert doch mit der Erfahrung, daß auch in der noch zu jungen, noch zu teilungsfreudigen Epidermis keine Spaltöffnungen entstehen können.

So stoßen wir auf Fragen, die für überall auftretende Probleme der pflanzlichen Gewebedifferenzierung wichtig sind.

Strasburger ${ }^{8}$ nannte die plasmareichen Initialen, aus denen die Spaltöffnungsschließzellen hervorgehen, Spezialmutterzellen. In Anlehnung an diese Bezeichnung möchten wir vorläufig ganz allgemein bei der pflanzlichen Gewebebildung Zellen, deren Bildung und Wirkung den Gesetzen gehorcht, die wir für die Spaltöffnungsmutterzellen feststellten, als Spezialzellen bezeichnen. Dieser Begriff sei noch etwas genauer umrissen: Eine Spezialzelle ist eine plasmareiche Zelle, die von einer plasmaärmeren abgetrennt wird, indem sich das Plásma vor der Teilung der Zelle lokal anhäuft und die neue Wand diese Plasmaanhäufung von der übrigen Zelle abgrenzt. Diese inäquale Teilung kann meist erst stattfinden, wenn die allgemeine Teilungsfähigkeit im Erlöschen begriffen ist. Häufig (oder immer?) ist die plasmareichere Zelle auch die kleinere; sie ist leistungsfähiger als die größere Nebenzelle. Die Spezialzelle kann weiterhin auch die Umgebung wieder zur Teilung anregen und dadurch Gewebe- oder Organanlagen entstehen lassen, an deren Bildung sich nicht nur die Spe- zialzelle selber beteiligt. Der gleiche teilungsfördernde Einfluß auf die Umgebung bedingt (weil hohe Teilungsfähigkeit ja jene inäqualen Teilungen verhindert), daß in der Nähe keine weiteren, sekundären Spezialzellen entstehen können; in einem größeren Gewebekomplex scheinen sich also die Initialen gegenseitig ,abzustoßen“, so daß die typischen Muster von Organanlagen usw. entstehen, die alle nicht den Charakter von Zufallsmustern tragen.

Hier soll skizzenhaft angedeutet werden, wie groß die Bedeutung der Bildung solcher Spezialzellen bei der pflanzlichen Gewebebildung ist.

Am Vegetationspunkt entstehen in einer gewissen Entfernung von der Spitze die Blattanlagen. Es sind mehrere Hypothesen darüber aufgestellt worden, welche Umstände hier einen Zellkomplex veranlassen, sich vorzuwölben. Eine ausführliche Diskussion soll bei späterer Gelegenheit erfolgen. Hier sei nur darauf hingewiesen, daß die Bildung einer Blattanlage damit beginnen kann, daß in einer Zelle eine Wand auftritt, die in anderer Richtung steht als die vorher eingeschobenen Wände. $\mathrm{Ob}$ es sich hierbei um eine typische inäquale Teilung wie bei der Bildung der Spaltöffnungsmutterzelle handelt, können wir noch nicht ganz sicher sagen, hoffen diese Frage jedoch bald zu klären. Mit dieser Deutung würde es jedenfalls wieder harmonieren, daß in der Umgebung dieser Ausgangszelle weitere Teilungen induziert werden, so daß also die Blattanlage nicht nur von einer Zelle aufgebaut wird, obwohl ihre Entstehung auf eine Zelle zurückgeht. Dieser teilungsfördernde Einfluß der Ausgangszelle auf die Umgebung bedingt, ebenso wie bei der Spaltöffnungsinitiale, daß in der Umgebung weitere Zellen dieser Art nicht auftreten können, diese vielmehr erst wieder in größerer Entfernung möglich sind, so daß das regelmäßige Muster von Blattanlagen entsteht. Die Bildung der einzelnen Blattanlage und des ganzen Musters von Blattanlagen können wir also wohl ebenso begreifen wie die Bildung einer Spaltöffnungsanlage mit den Nebenzellen und die Bildung des Spaltöffnungsmusters.

Im inneren Teil des Vegetationskegels ereignet sich offenbar etwas Ähnliches wie in der Epidermis bzw. Subepidermis. Es treten wieder, wenn die Entfernung vom Ort lebhafter Teilungen, also von der Spitze, ausreichend geworden ist, Teilungen auf, bei denen zum mindesten die Wand- 
richtung anders ist als bei den vorhergehenden Teilungen. Die so entstehenden Initialen stellen den Ursprung der Prokambiumstränge dar. Wieder gehen von ihnen Einflüsse aus, die in der Umgebung teilungsanregend wirken und so die Stränge dicker werden lassen. Gleichzeitig wird auch wieder verhindert, daß in der Umgebung weitere Initialen auftreten. So bildet sich das regelmäßige Muster der Gefäßbündel, die einen gleichmäßigen gegenseitigen Abstand einhalten.

Wenn in den heranwachsenden Blattanlagen die von den Initialen induzierte Teilungsfähigkeit
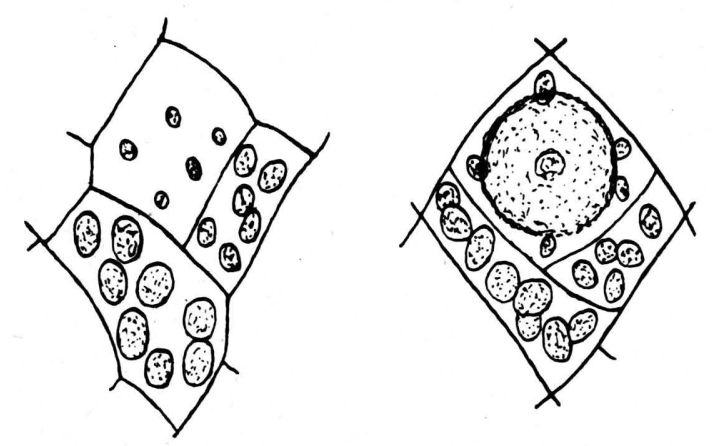

Abb. 21. Bildung der Chlorophyllzellen im Blatt von Sphagnum cymbifolium. Links in einem jüngeren, rechts in einem etwas älteren Stadium. Die Kerne der Chlorophyllzellen, rechts auch der Kern der künftigen Hyalinzelle, sind nicht eingezeichnet.

allmählich erlischt, entfällt auch die Hinderung der Bildung weiterer sekundärer Spezialzellen. So jedenfalls können wir es deuten, daß neue Teilungsnester entstehen, aus denen Blattlappen, Blattfiedern usw. hervorgehen. Jedoch müssen wir noch prüfen, ob hier wirklich wieder das gleiche Schema anwendbar ist, ob also diese Blattfiedern usw. auf eine durch inäquale Teilung entstandene Zelle zurückgehen. Schließlich jedenfalls wiederholt sich der Vorgang, wie wir auf den vorhergehenden Seiten ausführlich gezeigt haben, nochmals, und es bilden sich dabei die Spaltöffnungen, Haare usw.

Wenn der Hemmungsbereich einer Spezialzelle sehr klein ist, kann sich in jeder Zelle oder in jeder einzelnen einer größeren Anzahl untereinander der Herkunft nach gleichwertiger Zellen eine Spezialzelle bilden. Die so entstehenden Muster sind natürlich noch viel regelmäßiger. Beispiele dafür lassen sich leicht finden. Es sei etwa an die Bildung des Spaltöffnungsmusters bei Monokotylen erinnert, oder auch an die Wurzelhaarbildung bei vielen Pflanzen (die Wurzelhaar- bildung kann sich im einfachsten Fall so vollziehen, daß jede Epidermiszelle eine inäquale Teilung vollführt, nach der die plasmareichere Tochterzelle das Wurzelhaar bildet).

Aber auch in diesen Fällen ist die zur Initialenbildung führende inäquale Teilung wieder an die Voraussetzung geknüpft, daß die Teilungsfähigkeit im Erlöschen begriffen ist. Als ein schönes Beispiel sei hier noch auf die bekannte Differenzierung im Sphagnum-Blatt verwiesen. Die von der zweischneidigen Scheitelzelle abgetrennten Segmente teilen sich bekanntlich zunächst durch gewöhnliche Teilungen weiter auf. Dabei entstehen rhombische Zellen. Sind diese Teilungen beendet, so treten die inäqualen Teilungen auf, bei denen zwei kleinere Zellen, aus denen die Chlorophyllzellen werden, von der größeren Restzelle, die zur Hyalinzelle wird, abgetrennt werden.

Wir wollen hier nur noch folgendes zum Bekannten ergänzen: Die inäqualen Teilungen beginnen an der Spitze, obwohl hier die jüngsten Segmente liegen. Das hängt damit zusammen, daß an der breiter werdenden Basis noch länger gewöhnliche Teilungen stattfinden, die ja die inäqualen hindern. Vor der inäqualen Teilung legt sich der große Kern in den basalen Teil der rhombischen Zelle. Die neuen Wände folgen in ihrem Verlauf weitgehend der Kernoberfläche und sind infolgedessen gekrümmt. B a u e $\mathrm{r}^{11}$ fand bei der Untersuchung von Sphagnum-Blättchen: „In etwas älteren Blättchen, in denen sich eine Differenzierung der Zellen anbahnte, behielten die Chloroplasten der zukünftigen hyalinen Zellen ihre Größe bei, wurden aber kräftiger grün, während die Chloroplasten der Assimilationszellen mit dem Zellwachstum größer wurden und zugleich an Chlorophyllgehalt zunahmen." Nach den Untersuchungen in unserem Institut (Frau Göser) möchten wir dem hinzufügen, daß die Chloroplasten nicht erst mit dem Wachstum der werdenden Chlorophyllzellen größer werden; diese Größenzunahme beginnt vielmehr unmittelbar nach der Ausbildung der trennenden Wand. Die bloße Abgrenzung einer plasmareichen (aber nur einen kleinen Kern enthaltenden) Zelle von der plasmaärmeren (aber mit einem Riesenkern ausgerüsteten) Zelle genügt also, um die Plastiden rasch wachsen zu lassen. Die Plastiden der zuerst abge trennten Chlorophyllzelle haben daher auch einen deutlichen Vorsprung gegenüber denen der zweiten Chlorophyllzelle (Abb. 21).

Weitere Untersuchungen werden zu klären haben, ob den inäqualen Teilungen, denen offensichtlich eine große Bedeutung bei der pflanzlichen Differenzierung zufällt, noch mehr als eine quantitative Ungleichheit im verteilten Plasma zugrunde liegt.

11 L. B a u e r, Flora 136, 30 [1942]. 